TEMAS DE ACTUALIDAD Rev Chil Salud Pública 2014; Vol 18 (1): 25-32

\section{CULMINA EL PRIMER CICLO DE LA FORMACIÓN DE ESPECIALISTAS PARA LA ATENCIÓN PRIMARIAY EL SISTEMA PÚBLICO DE ATENCIÓN DE SALUD. ¿QUÉ HACER EN ADELANTE?}

TRAINING PROGRAM OF SPECIALISTS INCORPORATED IN THE PRIMARY HEALTH CARE TEAMS. At THE END OF THE FIRST SIX YEAR CYCLE, WHICH GOALS FOR THE NEXT STAGE?

\section{RESUMEN}

El Programa de formación de especialistas para la atención primaria de salud y el sistema público, fue iniciado por el Ministerio de Salud de Chile en el año 2007 y se implementa en ocho facultades de Medicina. El Programa dura seis años; la mitad del periodo transcurre en centros de salud familiar. La primera cohorte de médicos se graduará a comienzos del año 2014. Participan 498 médicos jóvenes, estudiando en hospitales de nivel universitario y trabajando en 73 centros de salud que atienden una población de cerca de dos millones de usuarios potenciales.

Actualmente hay suficiente evidencia de que las autoridades, los profesores de clinica, los residentes, las municipalidades y los evaluadores externos consideran que el Programa es necesario y efectivo. El Ministerio proporciona el financiamiento adecuado; pero todavía preocupa su estatus legal y organizacional.

Las actividades se orientan a la consolidación de la gestión clínica, al fortalecimiento de las funciones de promoción y prevención en los centros de salud, al progreso en la equidad y efectividad del sistema y a la educación de los usuarios y de las comunidades. Durante la formación también se desarrolla un curso (diploma) de Salud Pública.

Los objetivos de mayor alcance incluyen: la planificación de la cobertura a alcanzar durante el próximo ciclo de seis años, la definición de las condiciones deseables para ejecutar la atención primaria, y la evaluación del costoefectividad de las acciones, incluyendo el destino ulterior de los egresados.

Palabras clave: sistema de atención de salud, educación médica de posgrado, atención primaria de salud. 


\section{ABSTRACT}

The Training Program of specialists incorporated in the primary bealth care teams and in the public medical care system was started by the Ministry of Health of Chile in 2007 and is carried out in cooperation with eight Medical Faculties. The program lasts six years; half of the time is spent in primary care clinics. The fist cohort will graduate as specialists beginning of 2014. In all, 498 young physicians are now participating in the Program in 73 clinics that cover nearly 2 million potential users.

It is generally agreed by all concerned - authorities, faculty, residents, municipalities, external evaluators - that the Program is necessary and effective. However, at this stage and in spite of the financial and administrative support so far provided, there is a concern regarding the legal and organizational status of the Program.

Current efforts are being directed towards consolidating the clinical management of patients, strengthening promotion and prevention in the clinics, improving the equity and effectiveness of the system and educating the users and the communities. This is supported by a public health course delivered during the entire duration of the Program.

Broader objectives include: planning the coverage to be attained during the next six-year cycle; defining the required type of PHC facilities, and evaluating the cost-effectiveness of the Program, including the subsequent destination of its graduates.

Key words: health care system, postgraduate medical education, primary health care.

\section{LA SITUACIÓN DEL PROGRAMA DE FORMACIÓN DE ESPECIALISTAS EN EL MARCO DE LA ATENCIÓN PRIMARIA Y HOSPITALARIA DE LA SALUD EN CHILE, AÑO 2013.}

\subsection{El Programa}

El Programa ministerial de formación de especialistas al cual se refiere el título de este trabajo, se inició en el año 2007, por acuerdo entre el Ministerio de Salud y la Universidad de Chile (1-3).

El Programa contempla una formación de seis años, alternando la formación en los departamentos universitarios situados en los hospitales base de los servicios participantes, con rotaciones de trabajo asistencial y de salud pública en los centros de salud (consultorios) asignados. Las cuatro especialidades básicas incluidas en el programa son Medicina Interna, Pediatría, Obstetricia-Ginecología y Psiquiatría de Adultos. Recientemente han sido incorporadas Neurología, Psiquiatría Infantil y Oftalmología. Las remuneraciones de los médicos participantes, las de sus tutores, y los aranceles universitarios pertinentes son financiados por el Ministerio de Salud.

A su ejecución se han incorporado, a lo largo de los años 2008-2013, ocho facultades de Medicina acreditadas para formar especialistas; 19 campos clínicos hospitalarios, 24 comunas y 73 centros de salud (consultorios) en doce Servicios de Salud. Dichas facultades pertenecen a las universidades de Chile, de Valparaíso, de Concepción, de La Frontera, de Santiago de Chile, Austral de Chile, del Desarrollo y Diego Portales.

La población total inscrita en los centros de salud asciende a 1.901 .326 beneficiarios.

Al escribir este artículo, la cifra total de médicos que participan en este plan de formación es de 498, pertenecientes a seis cohortes (2008-2013), en siete especialidades: Medicina Interna, 179; Pediatría, 141; Ginecología-Obstetricia, 75; Psiquiatría de Adultos, 83; Psiquiatría Infantil y del Adolescente, 4; Neurología de Adultos, 1; y Oftalmología, 2. Ellos proceden de quince universidades chilenas y hay además un 
grupo reducido de residentes que provienen de universidades de Ecuador, Colombia y Bolivia.

El reforzamiento de la APS en los consultorios participantes se ve incrementado por un número de alrededor de 50 tutores clínicos y de salud pública y por la inclusión de uno o varios diplomados (por ejemplo, en Salud Pública) y otras actividades académicas.

El impacto del Programa al cual nos referiremos se logra por la actividad de los equipos de médicos participantes durante la fase sexenal de su formación como especialistas (4-15). Otra tarea será la de evaluar los efectos después de su egreso.

Este impacto inmediato en la atención de salud de los beneficiarios del sistema público ha sido reconocido por todas las contrapartes del Programa. Así lo expresaron los servicios de salud involucrados, en representación de las instituciones académicas y las municipalidades correspondientes, en la Jornada convocada por el subsecretario de Redes Asistenciales el 27 de marzo del 2013. El mismo juicio resultó de la evaluación externa realizada por un panel de expertos a fines del 2011 (17). Dicho impacto se ha verificado -en grado variable según las comunas y consultorios- en los siguientes aspectos: reintegración entre los niveles de la red; resolución en la primera línea de problemas de salud hasta entonces no abordados; resolución in situ de interconsultas pedidas por los médicos generales; reducción significativa de interconsultas no pertinentes a los niveles secundario y terciario del sistema; disminución de la solicitud de exámenes innecesarios; disminución de la prescripción de medicamentos superfluos; capacitación de los equipos de salud, incluyendo a los médicos generales de los consultorios; desarrollo a nivel comunitario de actividades de promoción y fomento de la salud y de prevención de las enfermedades más prevalentes; realización de estudios e investigaciones clínicas, epidemiológicas y operacionales que contribuyen a un mejor conocimiento de la situación de salud y de atención de salud de los beneficiarios cubiertos por el Programa.
Cabe destacar también el alto nivel académico de los médicos incorporados al Programa, como lo acreditan sus altas calificaciones de pregrado, las cuales, en el caso de los becarios de Medicina Interna de la Universidad de Chile del año 2013, ascienden a un promedio de 6,25 , y en el caso de los becarios de Psiquiatría de Adultos, a 6,35 (con un máximo de 7). Algunas de las muchas innovaciones y experiencias de trabajo en APS de los participantes quedan acreditadas en los libros de resúmenes de los trabajos presentados en las Jornadas Científicas del Programa realizadas los años 2011 y 2013 en la Facultad de Medicina de la Universidad de Chile (16). Trabajos y reuniones similares se realizan asimismo en las otras universidades participantes. Algunos de estos estudios e investigaciones han sido presentados en congresos y otras reuniones científicas nacionales e internacionales, y publicados en revistas científicas de las especialidades correspondientes (18-24).

En resumen, este Programa ha demostrado que los médicos formados en las universidades chilenas, así como procedentes de universidades extranjeras calificadas, están interesados en trabajar en los establecimientos de la primera línea de atención del Sistema Público de Salud, cuando se les ofrecen posibilidades de formación y desarrollo profesional y condiciones de trabajo apropiadas, favorables al progreso en sus aspiraciones en Medicina y a su crecimiento como personas.

Se aproxima el término de un primer "ciclo" de seis años en la vida del Programa. A comienzos de 2014, después de aprobar los exámenes pertinentes, recibirán su título de especialista los residentes de la primera cohorte, que ingresó en abril del año 2008.

Tanto el Programa como la atención primaria de salud del país, conocida muy de cerca a lo largo del desarrollo de este, han sido objeto de publicaciones que han ido dando cuenta detallada de su situación, aprendizajes y logros $(4,16)$. El presente trabajo es uno más de esta serie. 
Cuadro 1. Distribución de los EEF por universidades y campus clínicos.-población inscrita en los consultorios atendidos. Año 2013.

\begin{tabular}{|c|c|c|c|}
\hline UNIVERSIDAD & CAMPUS CLÍNICO & $N^{\circ}$ DE EEF & POBLACIÓN INSCRITA \\
\hline \multirow[t]{9}{*}{ De Chile } & Hosp. S. Juan de Dios & 97 & 357332 \\
\hline & Hosp. Barros Luco & 57 & 285117 \\
\hline & Hosp. González Cortés & 21 & $\because *$ \\
\hline & Hosp. Roberto del Río & 20 & $60233 * \%$ \\
\hline & Hosp. S. Borja Arriarán & 32 & 99660 \\
\hline & Hosp. del Salvador & 26 & 210099 \\
\hline & Hosp. Luis Tisné & 7 & $* *$ \\
\hline & Hosp. Calvo Mackenna & 14 & $\because *$ \\
\hline & Cl. Psiquiátrica Univ. & 8 & $\because *$ \\
\hline \multirow[t]{3}{*}{ De Santiago } & Hosp. S. José & 31 & 281688 \\
\hline & Hosp. Reg. de Rancagua & 4 & 35057 \\
\hline & Hosp. S. Borja Arriarán & 13 & 195945 \\
\hline De Valparaíso & Hosp. Van Buren & 26 & 170565 \\
\hline Del Desarrollo & Hosp. Alberto Hurtado & 62 & 230940 \\
\hline \multirow[t]{2}{*}{ De Concepción } & Hosp. Las Higueras & 38 & 142751 \\
\hline & Hosp. Grant Benavente & 6 & 60071 \\
\hline \multirow[t]{3}{*}{ Diego Portales } & Hosp. Psiquiátrico & 6 & 91061 \\
\hline & Hosp. Dipreca & 6 & \\
\hline & Hosp. Reg. de Rancagua & 5 & \\
\hline De la Frontera & Hosp. Dr. H. Henríquez & 14 & 70704 \\
\hline Austral de Chile & Hosp. Reg. de Valdivia & 12 & 47464 \\
\hline Total Programa & & 499 & 2338687 \\
\hline
\end{tabular}

* Hay población inscrita en otros Campus del Programa (se evita duplicar).

\subsection{Aspectos del Programa a considerar en la fase actual de su ejecución}

La iniciativa aborda un aspecto importante dentro de la estructura total del Sistema Público de Atención de Salud de Chile: la provisión de las atenciones médicas de promoción, prevención, curativas y de rehabilitación, tanto en la atención primaria como en los niveles ambulatorio y cerrado de los hospitales. A través de estas actividades, el Programa y sus participantes han logrado la progresiva reintegración de los profesionales médicos en el equipo de la "atención primaria” urbana y al mismo tiempo, han conseguido empezar a llenar el hiato entre los niveles del consultorio y del hospital, todo ello en beneficio de los usuarios y de la moral y eficacia laboral de los equipos multiprofesionales.
Esta iniciativa y las respectivas estrategias asistenciales y educacionales han recibido apoyo de las administraciones del sistema público en sus diversos niveles, incluyendo el nivel municipal, los servicios de salud, así como de las universidades y de los parlamentarios miembros de las Comisiones de Salud de la Cámara y del Senado. Parte importante de este análisis permanente es el funcionamiento de la Comisión Intercomunal, que involucra a representantes de la gestión de salud de varias de las comunas incluidas.

Sin embargo, puede comprobarse que la situación del Programa es todavía marginal en las estructuras del Ministerio de Salud y en las de algunas universidades. Existe el apoyo del ministro y del subsecretario de Redes Asistenciales con respecto al presupuesto y a las resoluciones administrativas básicas para el 
funcionamiento del Programa; pero la coordinación central del mismo carece del estatus y de los recursos de secretaría propios de una unidad formalmente estatuida (17).

Solo a causa del crédito logrado por el Programa y del apoyo trasversal mencionado, pudieron salvarse obstáculos derivados de que la legislación vigente no se ajusta a la racionalidad innovadora del esquema de formación y trabajo combinados en seis años. Estos obstáculos han constituido una especie de marginalidad jurídica que genera inseguridad a los participantes y que se ha resuelto, hasta el año 2013, mediante decisiones jurídico-políticas ad hoc.

Al comienzo de las actividades, también fue marginal el estatus de los especialistas en formación en algunos de los centros de salud municipalizados. Con todo, sus actividades, apoyadas por la Coordinación y por los docentes clínicos y de salud pública pertenecientes al Programa, han superado esta condición, la cual ha cambiado hacia un franco reconocimiento del aporte brindado.

La marginalidad que subsiste es tanto más injustificada y debería ser tanto más superable cuanto que la presencia de los "especialistas en formación" corresponde precisamente a lo que la población espera y exige: la atención de un médico que resuelva sus problemas de salud, con la posibilidad de sentir que cada cual tiene "su" médico, y a mayor abundamiento, que sea, en lo posible, un especialista o tenga el apoyo cercano de un especialista. Algunas de la promesas ofrecidas a la población bajo la forma del AUGE o GES (2004) están siendo parcialmente cumplidas, dentro de los límites de ese plan: acceso, oportunidad, protección financiera; lo que no se ha cumplido es la garantía de calidad en el sector público. Es precisamente para el logro de esta garantía esencial que el Programa de Formación de Especialistas para la APS muestra ya el camino.

\section{2. ¿QUÉ HACER EN ADELANTE?}

Como en todo emprendimiento social, se precisa tener una imagen de los objetivos que se quiere lograr. Se ha alcanzado ya un ja- lón muy importante; precisamos definir cuál debe ser el próximo, dentro de un proceso de avance por etapas, con perspectiva histórica.

2.1. Una tarea importante es la de consolidar la gestión clínica de los pacientes atendidos en el consultorio y, cuando ello proceda, también durante su atención en el nivel secundario o terciario, y en el domicilio. En este aspecto hay progreso y los médicos han visto cómo el mejoramiento del manejo clínico produce también mejoría de las condiciones para que dicho manejo exista. Algunos ejemplos: el autoagendamiento para el control del paciente por el mismo médico tratante; la supervisión de las referencias y contrarreferencias; el tiempo para la realización de "policlínicos" de especialidad y de "talleres de ingreso a programas" en el consultorio, con la colaboración de los especialistas tutores; persuasión constante relativa a la disponibilidad de medicamentos y exámenes esenciales, así como de boxes y equipos adecuados; la educación de la demanda; el cumplimiento de las guías, protocolos y otras normas. Respecto a este último punto, los médicos del programa deberán sentar el ejemplo de ser buenos clínicos, sabiendo que los instrumentos diagnósticos más efectivos son la anamnesis y el examen físico bien realizados; se precisa controlar el uso innecesario y hasta riesgoso del laboratorio, la imagenología, algunas pruebas y otras tecnologías. Del mismo orden de cosas será el control de la informatización "no médica” de la historia clínica (ficha), que puede entorpecer el contacto médico-paciente y el estudio de los casos y de las series clínicas. El computador es útil, pero debe estar al servicio del médico y del paciente y no a la inversa.

Estas prácticas fortalecerán el concepto y el ejercicio de lo que es el "trabajo médico" y la "atención de salud", de modo que no se produzca un desequilibrio respecto a otros esquemas de pensamiento y acción también importantes, como la epidemiología, la administración y la economía.

2.2. Otro aspecto a perfeccionar en el futuro inmediato es la superación de las ineficiencias administrativas que afectan al sistema y, en particular, a la atención primaria. No basta con identificarlas y criticarlas. Se las 
deberá analizar para conocer sus factores e ir corrigiéndolos progresivamente.

Por un efecto de retroalimentación podrá fortalecerse la buena administración de los centros de salud, de las direcciones municipales y de las direcciones de servicios de salud. Un aspecto pertinente es el conocimiento y la concordancia de todo el equipo de cada centro de salud respecto de los protocolos de las actividades en que se participa.

Las experiencias de los especialistas en formación (EEF) en el trabajo en equipo y en la capacitación de sus diversos integrantes (incluidos ellos mismos) apuntan a la posibilidad de orientar la planificación, la formación y la administración del personal ("recurso humano"). Las mismas experiencias deberán contribuir a la programación y organización de la provisión local de la atención, y al diseño de un esquema de información pertinente, oportuno, viable y aprovechable por todos, para administrar y para investigar.

2.3. En el lugar donde estén como proveedores o ejecutores de actividades y programas asistenciales, preventivos y promotores de salud, los EEF son una palanca poderosa para la equidad y efectividad del sistema. Equidad y efectividad, ¿en qué? En el logro parejo de mejoramiento en el estado de salud, donde el control y la disminución de problemas prevalentes como la obesidad, la "depresión”, el alcoholismo, el tabaquismo, la tuberculosis, las enfermedades tiroideas, los cánceres del cuello uterino y de la mama, los reumatismos y las complicaciones de hipertensión arterial y de diabetes, son objetivos evidentes y alcanzables.

Para lograr una real cobertura de estos problemas se requiere: a) extender el horario de atención hasta las 20 horas, ajustando las agendas del personal necesario y contratando personal adicional si tales ajustes no resultan ser suficientes; b) obtener en los consultorios las capacidades de ejecución de procedimientos de necesidad frecuente, tales como ecografías abdominales y obstétricas, cirugía menor, oftalmología y otorrinolaringología de nivel primario; y c) disponer continuamente de los medicamentos y exámenes esenciales (requisito que en algunas municipalidades o cor- poraciones sí se cumple gracias a una buena gestión).

Sabemos que el efecto de equidad y efectividad en la satisfacción de la población y de los usuarios con el sistema de atención en que somos actores está mejorando. Pero es necesario presentar un sostén cuantitativo, para convencer y para afinar el rumbo hacia su consecución plena.

2.4. También debe ser objetivo de la etapa próxima el lograr una educación de los usuarios y de la población general de los sectores asignados, para que comprendan el sistema de atención, su sentido como parte de la seguridad social y del desarrollo, y que sepan cómo se programan, se financian y organizan las actividades. Durante su rotación por consultorio, los EEF deben dedicar cada vez más atención al trabajo con la comunidad, con los colectivos existentes en cada sector. Es muy importante que refuercen esta vertiente, donde los médicos han estado ausentes. Hay que elaborar los protocolos y agendas de esta actividad en momentos precisos del sexenio. Se logrará así hacer realidad ese otro principio de la atención de salud: la participación activa de la sociedad.

2.5. Nos hemos referido a proveedores y usuarios. Hay que obtener que la cabeza del sistema siga asumiendo la sustentación del Programa y monitoreando su marcha, como lo ha hecho desde 2007 hasta ahora; que lo institucionalice con mayor formalidad en los aspectos organizacionales y legales. Para ello hay que seguir demostrando, con documentación, publicaciones y pruebas consistentes, con investigación, con el apoyo de los métodos sociológicos y epidemiológicos, la efectividad de los EEF en generar salud física y mental, y en producir satisfacción poblacional.

\section{DESARROLLO DEL PROGRAMA A NIVEL INSTITUCIONAL}

3.1. Para corregir la marginalidad jurídica del Programa, el ministerio deberá enviar al Parlamento un proyecto por el cual se introduzca en una de las leyes médicas (15.076 o 19.664), 
una disposición que legalice esta modalidad de formación y destinación, la cual ha sido definida en resoluciones del propio ministerio. En tal disposición debería explicitarse la calidad de funcionarios del sistema, de los médicos que se forman y trabajan en el Programa. Recientemente se formó, por decisión de las autoridades superiores del ministerio, una comisión que preparó una propuesta de la reforma legal necesaria.

3.2. Para definir un horizonte claro del compromiso nacional implicado por el Programa, se deberá elaborar el plan de cobertura poblacional y territorial a alcanzar a lo largo del segundo sexenio. Ello requiere contar con un diagnóstico suficientemente preciso de la realidad de la atención primaria, sabiendo que ella es diferente en la región Metropolitana respecto de las demás regiones. Esa cobertura debería agregar, en la región Metropolitana, dos centros de salud en cada una de las siguientes comunas: Renca, Conchalí, Huechuraba, San Miguel, El Bosque, Quilicura, Quinta Normal y Maipú. Es prioritario incluir Antofagasta, para lo cual debe asegurarse la acreditación de la universidad correspondiente en cuanto a la formación de los especialistas básicos. Las regiones del Biobío, La Araucanía, Los Ríos y Valparaíso, deberán examinar este tema junto con la Coordinación Nacional del Programa.

Los nuevos centros de salud que se están creando deben beneficiarse de la experiencia adquirida respecto al tamaño y a las facilidades que se necesitan para el cumplimiento de sus objetivos asistenciales y docentes.

Además, el ministerio debería estudiar y evaluar la composición de los equipos de sector de los centros y el rol de las diversas profesiones en ellos, incluyendo el de los médicos generales, el de los médicos de familia, y el de los especialistas en formación (EEF), para adecuarlos a las necesidades cambiantes de la población y del Sistema.

3.3. En el aspecto financiero, se deberá medir y evaluar anualmente el costo-efecto del Programa, definiendo como efecto -en una primera fase- la cantidad de personas a la cual alcanzará en forma directa o indirecta la influencia de la acción de los EEF, por en- contrarse un equipo de estos en el centro de salud que les corresponde por su residencia. El costo se definirá como el monto de dinero incluido en el ítem respectivo del presupuesto devengado anual del Ministerio de Salud.

El nivel central y los servicios de salud deberán velar porque las asignaciones presupuestarias per capita y de los programas específicos de la APS lleguen oportunamente a las comunas, e igualmente, porque el uso de estos recursos sea eficiente.

3.4. Será indispensable recoger, a partir de 2014, la información completa acerca del destino de los especialistas titulados en el Programa. Ello será importante para seguir evaluando el efecto del mismo en la realidad de la atención de salud, en sus distintos niveles y en las diversas regiones del país.

\section{REFERENCIAS}

1. Ministerio de Salud, Departamento de Formación y Capacitación de Recursos Humanos, Coordinación Nacional del Programa de Formación de Especialistas para la Atención Primaria Urbana (CN-PFE-APS). "Programa de formación de Médicos en Especialidades Básicas para la APS Urbana en el Sistema Público de Atención de Salud", Santiago, Chile, 2009.

2. Ministerio de Salud, Subsecretaría de Redes Asistenciales, Departamento de Formación y Capacitación. Resolución Exenta del 8 de marzo de 2010 que aprueba el texto del Programa de Formación de Médicos en Especialidades Básicas para la Atención Primaria en el Sistema Público de Atención de Salud.

3. Ministro de Salud. Incorporación de tutores médicos al Programa de Formación de Especialistas Básicos, para mejoramiento de la resolutividad en Consultorios Generales Urbanos de Atención Primaria de Salud, Oficio C 32/2632 de 17 de agosto 2009.

4. Montoya-Aguilar, C. Una experiencia en medicina integral: informe preliminar, Cuad Med Soc (Chile) 1961; 2(3): 10-13.

5. Montoya-Aguilar C. Aspectos de la formación Médica en Chile. Su relación con los conceptos de medicina integral y medicina 
familiar y con la planificación de recursos humanos para el sistema de salud, Cuad Med Soc (Chile) 1998; 39(2): 42-50.

6. Ipinza M. Cómo solucionar el déficit de médicos en consultorios urbanos municipalizados. Cuad Med Soc (Chile) 2004; 44(2): 73-80.

7. Ipinza M. Calidad y atención integral de salud, Cuad Med Soc (Chile) 2007; 47(1): 5-17.

8. Montoya-Aguilar C. Alma Ata otra vez y la experiencia de Chile, Cuad Med Soc (Chile) 2008; 48(3): 147-154.

9. Montoya-Aguilar C, Ipinza M. Un programa para recuperar y mejorar el aporte de los médicos en la atención primaria de salud, Cuad Med Soc (Chile) 2009; 49(1): 16-25.

10. Vargas N, Tassara R, Domínguez M, Puxant M, Mendoza J J, Faúndez R. Programa de especialistas para la atención primaria: experiencia y visión de académicos de la Universidad de Chile al primer año de marcha. Cuad Med Soc (Chile) 2009; 49(2): 87-93.

11. Coordinación General del Programa de Formación de Especialistas Básicos para la APS urbana, Anexo 1 al Convenio entre Servicios de Salud y Municipalidades participantes, Santiago 2009.

12. Ipinza M. El modelo de atención integral, base doctrinaria del programa de formación de médicos especialistas básicos para la atención primaria de salud. Cuad Med Soc (Chile) 2010; 50(2): 132-142.

13. Montoya-Aguilar C, Ipinza M. La atención primaria en Chile y el papel de los médicos. Nuevos objetivos en el año 2010, Cuad Med Soc (Chile) 2010; 50(3): 167-177.

14. Montoya-Aguilar C, Vargas N. Organización de la atención primaria de salud urbana, con incorporación de especialistas en formación, Cuad Med Soc (Chile) 2011; 51(1): 40-47.

15. Programa de Formación de Especialistas para la APS, Jornadas de investigación, 2011 y 2013, Libros de Resúmenes.
16. Departamento de Formación, Capacitación y Estudios, División de Gestión y Desarrollo de las Personas, Subsecretaría de Redes Asistenciales, "Evaluación del Programa de formación de especialistas básicos en la atención Primaria de Salud”, diciembre de 2011.

17. Aravena, P, Ruiz P, Experiencia en la implementación de un policlínico de choque en salud mental en un establecimiento de atención primaria, Cuad Med Soc (Chile) 2011; $51(1): 5-15$.

18. Maureira, MJ. Prevención secundaria de eventos cardiovasculares con el uso de estatinas. (Hospital Barros Luco Trudeau y Cesfam A. Neghme, Comuna Pedro Aguirre Cerda). Presentación a las Primeras Jornadas Científicas del Programa de Formación de especialistas para la APS urbana, septiembre 2011.

19. Maureira, MJ, Sepúlveda T. Mejorando la resolutividad de reumatología en la Atención Primaria de Salud (Unidad de Reumatología del Hospital Barros Luco Trudeau, Centro de Rehabilitación Comunal y Cesfam A. Neghme, Comuna Pedro Aguirre Cerda), Presentación al $28^{\circ}$ Congreso Çhileno de Reumatología, octubre 2011.

20. Díaz C, Picó Y, Glisser M. Utilización de gráficos de crecimiento infantil, Cuad Med Soc (Chile) 2012; 52(1): 6-13.

21. Morales F. Horas de recetas: cómo atender a una ficha, Cuad Med Soc (Chile) 2012; 52(2): 27-30.

22. Vargas NA, Gómez G. Programa de Especialistas para la Atención Primaria, Ministerio de Salud: adherencia al programa en sus primeros cuatro años de funcionamiento. Rev Hosp Clin Univ Chile 2012; 23: 277-82.

23. Barragán T, Glisser M. Encuestas e indicadores de duración de lactancia materna en tres Consultorios del Área Sur de Santiago. Presentación al Congreso de la Sociedad Chilena de Pediatría, noviembre 2013 (Tercer Premio del Congreso). 